\title{
ON THE CANONICAL SUBSTITUTION IN THE HAMILTON-JACOBI CANONICAL SYSTEM OF DIFFERENTIAL EQUATIONS.
}

BY DR. D. C. GILLESPIE.

(Read before the American Mathematical Society, September 5, 1907.)

LIE established a one-to-one correspondence between the integrals of the canonical system of differential equations and the one-parameter continuous groups of contact transformations of which the system admits, $i$. e., making use of an integral of the system one can construct the infinitesimal transformation of a group of which the system admits, or, on the other hand by making use of the infinitesimal transformation of a group of which the system admits one can construct an integral of the system.* This theorem is the foundation of the modern transformation theory of dynamical systems. $\uparrow$ The single canonical substitution (introduced by Jacobi) is of importance in the transformation and simplification of the dynamical equations.

The purpose of this paper is to define a type of the single canonical substitution which leads to an integral of the equations, $i . e$., if one knows a member of the defined type of canonical substitutions one can construct an integral of the system using only algebraic operations.

A system of differential equations which has the form

(1) $\frac{d \pi}{d x}=\frac{\partial H}{\partial y}, \quad \frac{d y}{d x}=-\frac{\partial H}{\partial \pi}, \quad \frac{d \kappa}{d x}=\frac{\partial H}{\partial z}, \quad \frac{d z}{d x}=-\frac{\partial H}{\partial \kappa}$

is called a canonical system. $H$ is a known function of $y, \pi$, $z, \kappa$ and $x ; y, \pi, z$ and $\kappa$ are the unknown functions; thus the solution of the system of four equations (1) consists in determining the four unknowns $y, \pi, z$ and $\kappa$ as such functions of $x$ that the equations become identities in $x$. The functions $y$ and $\pi$, as also $z$ and $\kappa$, are called conjugate.

A substitution which leaves the form of the system (1) unchanged, though the function $H$ may or may not be changed, is a canonical substitution.

\footnotetext{
* Whittaker, Analytical Dynamics, page 308.

† Ibid., page 292.

† Poincaré, Mécanique céleste ; Jacobi, Vorlesungen über Dynamik.
} 
In order to avoid complicated formulas I take a system of four equations. The generalization of the theory from four to $2 n$ equations is evident.

The system of equations (1) expresses the necessary condition that the integral

$$
\int_{1}^{2}\left\{\pi y^{\prime}+\kappa z^{\prime}+H(y, \pi, z, \kappa, x)\right\} d x
$$

(where $d y / d x \equiv y^{\prime}, d z / d x \equiv z^{\prime}$, and 1 and 2 stand for any limits of integration) take a maximum or a minimum value; $i$. e., if the question be, what functions of $x$ must $y, \pi, z$ and $\kappa$ be in order that the integral (2) shall take an extreme value, the answer is, only such functions as satisfy the equations (1).

In order that $y$ and $z$ be such functions of $x$ that the integral

$$
\int_{1}^{2} F\left(y^{\prime}, y, z^{\prime}, z, x\right) d x
$$

take an extreme value, it is necessary that $y$ and $z$ satisfy the system of equations

If now

$$
\frac{d}{d x}\left(\frac{\partial F}{\partial y^{\prime}}\right)-\frac{\partial F}{\partial y}=0, \frac{d}{d x}\left(\frac{\partial F}{\partial z^{\prime}}\right)-\frac{\partial F^{\prime}}{\partial z}=0 .
$$

$$
F\left(y^{\prime}, y, z^{\prime}, z, x\right) \equiv H(y, \pi, z, \kappa, x)+y^{\prime} \pi+z^{\prime} \kappa,
$$

whereby is understood that instead of $\pi$ and $\kappa$ are substituted their values in terms of $y^{\prime}, y, z^{\prime}$, and $z$, determined from the equations

(6) $y^{\prime}=-\frac{\partial H(y, \pi, z, \kappa, x)}{\partial \pi}, \quad z^{\prime}=-\frac{\partial H(y \cdot \pi, z, \kappa, x)}{\partial \kappa}$,

then the two systems (4) and (1) are equivalent. In fact let

$$
\begin{aligned}
& y=y\left(x, c_{1}, c_{2}, c_{3}, c_{4}\right), \quad z=z\left(x, c_{1}, c_{2}, c_{3}, c_{4}\right), \\
& \pi=\pi\left(x, c_{1}, c_{2}, c_{3}, c_{4}\right), \quad \kappa=\kappa\left(x, c_{1}, c_{2}, c_{3}, c_{4}\right)
\end{aligned}
$$

$\left(c_{1}, c_{2}, c_{3}, c_{4}\right.$ are arbitrary constants) be the complete solution of $(1)$, then

$$
y=y\left(x, c_{1}, c_{2}, c_{3}, c_{4}\right), \quad z=z\left(x, c_{1}, c_{2}, c_{3}, c_{4}\right)
$$


is the complete solution of (4). Both systems of equations are satisfied by the same values of $y$ and $z$. Conversely now let

$$
y=y\left(x, c_{1}, c_{2}, c_{3}, c_{4}\right), \quad z=z\left(x, c_{1}, c_{2}, c_{3}, c_{4}\right)
$$

be the complete solution of $(1)$, then

$$
\begin{gathered}
y=y\left(x, c_{1}, c_{2}, c_{3}, c_{4}\right), \quad z=z\left(x, c_{1}, c_{2}, c_{3}, c_{4}\right), \\
\pi=\frac{\partial}{\partial y^{\prime}} F\left(y^{\prime}, y, z^{\prime}, z, x\right), \quad \kappa=\frac{\partial}{\partial z^{\prime}} F\left(y^{\prime}, y, z^{\prime}, z, x\right)
\end{gathered}
$$

is the complete solution of (4). Thus the problems of integrating these two systems of equations are equivalent.

We will now suppose a canonical substitution known for the system of differential equations (4),

$$
\begin{gathered}
\pi_{1}=f_{1}(y, \pi, z, \kappa, x), \kappa_{1}=f_{2}(y, \pi, z, \kappa, x), z_{1}=f_{3}(y, \pi, z, \kappa, x) \\
y_{1}=f_{4}(y, \pi, z, \kappa, x), \quad x_{1}=f_{5}(y, \pi, z, \kappa, x)
\end{gathered}
$$

Under this substitution the $H(y, \pi, z, \kappa, x)$ goes over into another function $H^{*}\left(y_{1}, \pi_{1}, z_{1}, \kappa_{1}, x_{1}\right)$, but the form of the equations remains canonical. We wish to impose a condition that will fix the substitution (10) as a member of the type of canonical substitutions which leads to an integral of (4). The condition is that the $y$ and $z$ functions of the independent variable $x$ remain unchanged. This means that if

$$
\begin{aligned}
& y=\phi_{1}\left(x, c_{1}, c_{2}, c_{3}, c_{4}\right), \quad z=\phi_{2}\left(x, c_{1}, c_{2}, c_{3}, c_{4}\right), \\
& \quad \pi=\phi_{3}\left(x, c_{1}, c_{2}, c_{3}, c_{4}\right), \quad \kappa=\phi_{4}\left(x, c_{1}, c_{2}, c_{3}, c_{4}\right)
\end{aligned}
$$

is the complete solution of (4), then

$$
\begin{aligned}
& y_{1}=\phi_{1}\left(x_{1}, c_{1}, c_{2}, c_{3}, c_{4}\right), \quad z_{1}=\phi_{2}\left(x_{1}, c_{1}, c_{2}, c_{3}, c_{4}\right), \\
& \pi_{1}=\omega_{1}\left(x_{1}, c_{1}, c_{2}, c_{3}, c_{4}\right), \quad \kappa_{1}=\omega_{2}\left(x_{1}, c_{1}, c_{2}, c_{3}, c_{4}\right)
\end{aligned}
$$

(where $\omega_{1}$ and $\omega_{2}$ mean simply functions different from $\phi_{3}$ and $\left.\phi_{4}\right)$ is the complete solution of the system of equations obtained by subjecting the system (1) to the substitution (10).

In order to determine whether a given canonical substitution belongs to the defined type, it would seem necessary to know 
the complete solutions both of the system of equations (1) and the system obtained by subjecting (1) to the given substitution. This however is not the case. For suppose again that (10) is any given canonical substitution of which we wish to determine whether it belongs to the defined type. Transforming the system of equations (1) by (10), we obtain a new function

$$
H^{*}\left(y_{1}, \pi_{1}, z_{1}, \kappa_{1}, x\right)
$$

and hence a new system of differential equations

$$
\begin{array}{ll}
\frac{d \pi_{1}}{d x_{1}}=\frac{\partial H^{*}}{\partial y_{1}}, & \frac{d \kappa_{1}}{d x_{1}}=\frac{\partial H^{*}}{\partial z_{1}}, \\
\frac{d y_{1}}{d x_{1}}=-\frac{\partial H^{*}}{\partial \pi_{1}}, & \frac{d z_{1}}{d x_{1}}=-\frac{\partial H^{*}}{\partial \kappa_{1}} .
\end{array}
$$

Making use of this new function $H^{*}\left(y_{1}, \pi_{1}, z_{1}, \kappa_{1}, x_{1}\right)$, we form a new function $F^{*}\left(y_{1}^{\prime}, y_{1}, z_{1}^{\prime}, z_{1}, x_{1}\right)$, just as in (5),

$$
F^{*}\left(y_{1}^{\prime}, y_{1}, z_{1}^{\prime}, z_{1}, x\right) \equiv H^{*}\left(y_{1}, \pi_{1}, z_{1}, \kappa_{1}, x_{1}\right)+y_{1}^{\prime} \pi_{1}+z_{1}^{\prime} \kappa,
$$

where instead of $\pi_{1}$ and $\kappa_{1}$ are substituted their values in terms of $y_{1}^{\prime}, y_{1}, z_{1}^{\prime}$ and $z_{1}$ determined from the equations

$$
y_{1}^{\prime}=-\frac{\partial H^{*}\left(y_{1}, \pi_{1}, z_{1}, \kappa_{1}, x_{1}\right)}{\partial \pi_{1}}, \quad z_{1}^{\prime}=-\frac{\partial H^{*}\left(y_{1}, \pi_{1}, z_{1}, \kappa_{1}, x_{1}\right)}{\partial \kappa_{1}} .
$$

We express now the necessary condition that the integral

$$
\int_{1}^{2} F^{*}\left(y_{1}^{\prime}, y_{1}, z_{1}^{\prime}, z_{1}, x_{1}\right) d x_{1}
$$

take an extreme value and obtain the equations

$$
\frac{d}{d x_{1}}\left(\frac{\partial F^{*}}{\partial y_{1}^{\prime}}\right)-\frac{\partial F^{*}}{\partial y_{1}}=0, \quad \frac{d}{d x_{1}}\left(\frac{\partial F^{*}}{\partial z_{1}^{\prime}}\right)-\frac{\partial F^{*}}{\partial z_{1}}=0 .
$$

The systems of equations (1) and (4) are satisfied by the same functional values of $y$ and $z, y=f_{1}\left(x, c_{1}, c_{2}, c_{3}, c_{4}\right), z=f_{2}\left(x, c_{1}, c_{2}, c_{3}\right.$, $\left.c_{4}\right)$; so also are the systems (11) and (13), $y_{1}=f_{1}^{*}\left(x_{1}, c_{1}, c_{2}, c_{3}, c_{4}\right)$, $z_{1}=f_{2}^{*}\left(x_{1}, c_{1}, c_{2}, c_{3}, c_{4}\right)$. If now the transformation (10) belongs to the defined type, then $f_{1}\left(x, c_{1}, c_{2}, c_{3}, c_{4}\right) \equiv f_{1}^{*}\left(x, c_{1}, c_{2}, c_{3}, c_{4}\right)$ and 
$f_{2}\left(x, c_{1}, c_{2}, c_{3}, c_{4}\right) \equiv f_{2}^{*}\left(x, c_{1}, c_{2}, c_{3}, c_{4}\right)$. This means of course that if we drop the subscripts from $x_{1}, y_{1}, z_{1}$ in the system (13), it becomes identical with (4). This then is the condition that a given transformation be of the defined type.

Since the system of equations (4) expresses the necessary condition that both the integrals

$$
\int_{1}^{2} F\left(y^{\prime}, y, z^{\prime}, z, x\right) d x, \quad \int_{1}^{2} F^{*}\left(y^{\prime}, y, z^{\prime}, z, x\right) d x
$$

take an extreme value,*

$$
\left|\begin{array}{ll}
F_{y^{\prime} y^{\prime}} & F_{y^{\prime} z^{\prime}} \\
F_{z^{\prime} y^{\prime}} & F_{z^{\prime} z^{\prime}}
\end{array}\right| \div\left|\begin{array}{cc}
F_{y^{\prime} y^{\prime}}^{*} & F_{y^{\prime} z^{\prime}}^{*} \\
F_{z^{\prime} y^{\prime}}^{*} & F_{z^{\prime} z^{\prime}}^{*}
\end{array}\right|=\text { const. }\left(F_{y y^{\prime}} \equiv \frac{\partial^{2} F}{\partial y^{2}}, \text { etc. }\right)
$$

is an integral of the system (4). This, as we have seen, is equivalent to the construction of an integral of the system (1).

Example. As a simple example where the systems consist of only two equations, we take

$$
\frac{d \pi}{d x}=0, \quad \frac{d y}{d x}=-\pi
$$

here $H \equiv \frac{1}{2} \pi^{2}, F \equiv H+y^{\prime} \pi \equiv \frac{1}{2} \pi^{2}+y^{\prime} \pi$, or since by (1) $y^{\prime}=-\pi, F \equiv-\frac{1}{2} y^{\prime 2}$. The integral $\int_{1}^{2}-\frac{1}{2} y^{\prime} d x$ has as necessary condition for an extreme value

$$
y^{\prime \prime}=0 \text {. }
$$

We subject (1) to the substitution

$$
x=y_{1}, \quad y=-x_{1}, \quad \pi=\pi_{1}
$$

and obtain

$$
\frac{d \pi_{1}}{d x_{1}}=0, \quad \frac{d y_{1}}{d x_{1}}=-\frac{1}{\pi_{1}},
$$

$H^{*}=\log \pi_{1}, F^{*}=\log \pi_{1}+y^{\prime} \pi_{1}=\log \left(-1 / y_{1}^{\prime}\right)-1$, the necessary

*D. C. Gillespie, "On the construction of an integral of Lagrange's equations in the calculus of variations," Bulletin, $2 d$ series, vol. 13, no. 7, pp. 345-348. 
condition that the integral $\int_{1}^{2}\left(\log \left(-1 / y_{1}^{\prime}\right)-1\right) d x$ take an extreme value is

$$
y_{1}^{\prime \prime}=0
$$

the substitution (3) is a member of the defined type and $F_{y^{\prime} y^{\prime}} \div F_{y y^{\prime}} \equiv y^{\prime}=$ const. is an integral of $(2) . y^{\prime}=c$, $\pi=\partial F^{\prime} \partial y^{\prime}=-y^{\prime}$ gives $\pi=-c$ for an integral of $(1)$.

CoRNell UNIVERSITY, August, 1907.

\section{THE MAXIMUM VALUE OF A DETERMINANT.}

BY DR. F. R. SHARPE.

HADAMARD * has shown that the maximum value of a determinant when the absolute value of each element does not exceed 1 is $n^{\frac{1}{2} n}$. The square of such a maximum determinant is a determinant having all its elements 0 except those of the principal diagonal. If the elements are restricted to real values, they are each \pm 1 and are so arranged that when compared row with row there is always an equal number of changes and permanences of sign amongst the corresponding elements. Hence $n$ is necessarily even. If we compare any two rows with a third row, the division of changes and permanences is again even. Hence $n$ must be a multiple of 4 . By a rearrangement of signs and order of columns we can always arrange any three rows in the form which for the case of $n=12$ is

$$
\begin{array}{rrrrrrrrrrrr}
1 & 1 & 1 & 1 & 1 & 1 & 1 & 1 & 1 & 1 & 1 & 1 \\
1 & 1 & 1 & 1 & 1 & 1 & -1 & -1 & -1 & -1 & -1 & -1 \\
1 & 1 & 1 & -1 & -1 & -1 & 1 & 1 & 1 & -1 & -1 & -1
\end{array}
$$

The actual maximum determinant is known for the following cases: (1) $n$ a power of 2 , (2) $n=12$ or 20 , (3) when the factors of $n$ are any of the preceding numbers. For example, when $n$ is 8 , the determinant is

* Bull. des Sciences math., 1893. 